\title{
Global Consciousness (Cognitivism to Connectivism) \& Better Worldliness-Data Study of Spiritual Consciousness Measurement
}

\author{
Swati Idnani ${ }^{1}$, Sneha Idnani ${ }^{2}$ and Suresh Idnani ${ }^{3}$ \\ 1. Cognizant Technology Solutions, Based Out of Hartford, CT, USA \\ 2. Tata Consultancy Services, Based Out of Gurgaon, Haryana, India \\ 3. Indian Bank (Retired), Based Out of Connaught Place, New Delhi, India
}

\begin{abstract}
Our individual minds, though distinct and uniquely ours, may also join with others in a kind of mental symphony that now and then becomes audible against a prevailing background of static. That's a conclusion suggested by the Global Consciousness Project (GCP). After many years of monitoring multiple world events, researchers report strong evidence of some kind of transpersonal mentality that seems to emerge when many people share a common concern or experience. At such times, a global network of devices employing quantum tunneling has found weak but definite signs of coherence arising out of background "noise" or randomness. As propounded time and again by Prof. P. S. Satsangi Sahab— the 8th spiritual leader of Radhasoami faith, "In human frame, the spirit force resides in the innermost core and is thus covered by subtle mind and gross matter. Accordingly, there is spiritual emanation in the form of spiritual wave, particularly from the eyes and forehead of a human being”. He has also gone on to state- “As implied by the fundamental axiom of spiritual consciousness, the spirit force possesses prime energy which evolves into various mental or physical forces by its association with media of different kinds”. This, therefore can be measured-as suggested by various experiments conducted as part of Global consciousness project as well as a preliminary systemic study of spiritual consciousness of Dayalbagh community, during congregational meditation-cum-prayer meetings in the community Hall and measured via random number generators. Through our topic, we aim to focus on conducting a data science specific study of GCP/systemic experiments that allude to the view that there is no disparity between physical science and spiritual science Extend the conclusions to whether Better worldliness can be achieved by raising collective spiritual consciousness.
\end{abstract}

Key words: Global consciousness, noise, spiritual science, randomness.

\section{Introduction}

Our individual minds, though distinct and uniquely ours, may also join with others in a kind of mental symphony that now and then becomes audible against a prevailing background of static.

Experiments around the world have been geared towards establishing if we as human beings are isolated islands of consciousness or are we more interconnected than we think we are.

Corresponding author: Swati Idnani, B.E. (Electronics)/PGDBA (Finance), research fields: global consciousness and better worldliness/consciousness studies.

\section{Objectives}

Research based conclusions towards:

GCP/systemic experiments that allude to the view that there is no disparity between physical science and spiritual science;

Extend the conclusions to whether Better worldliness can be achieved by raising collective spiritual consciousness.

\section{Experiments}

3.1 Around the World

Global consciousness (sometimes called “field 


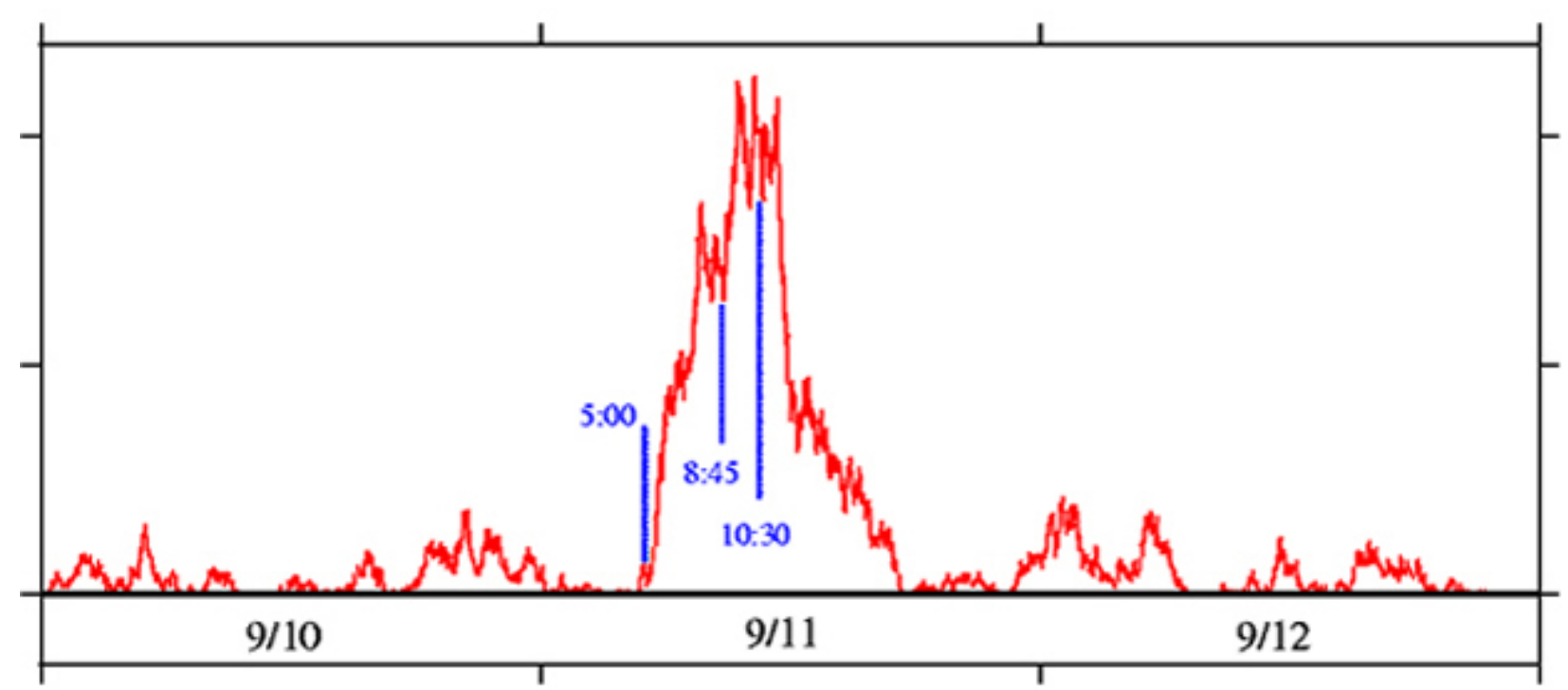

Fig. 1 Random number generator output during 9/11/2001 attacks.

consciousness") is the notion that when groups of people focus their minds on the same thing, they influence "the world at large" (Radin 1997: ch. 10).

Most of the world research has been focused on a detection system consisting of a global network of Random Number Generators (RNGs) based on Quantum tunneling.

Each RNG outputs a continuous stream of completely unpredictable zeroes and ones. The stream ordinarily averages out at $50 \%$ ones and $50 \%$ zeros, just as flipping coins tends to produce roughly equal heads and tails over time. The RNG data are transmitted to a central archive for later analysis.

When events engage millions of minds and hearts at once, structure seems to emerge out of what would otherwise be randomness. The global network departs a bit from the normal generation of random ones and zeros. The RNGs' behaviors become slightly correlated and the system as a whole appears to shift toward coherence.

\subsection{DEI Research Efforts in Kalpakkam}

Via the use of MEG (Magneto-Encephalogram as against EEG for sensitive measurements), which does not disturb the meditation process-it's many sensors have been used to pick up signals in the brain or its nearby environment. Experiments have been done to record consciousness elevation while listening to various sounds.

In the Atomic Research Centre at Kalpakkam, experimentation in properly shielded chambers were done showing that group meditation as well as individual brought up some of the frequencies of interest i.e. 4.5, 76, 108 and $126 \mathrm{~Hz}$ repeatedly.

\section{Conclusions}

As discovered in the experiments conducted through detection systems, and established by Most Revered Prof. P.S. SatsangiSahab as a preferred future course of research \& experimentation-it may be prudent to explore in the direction of measuring influence of meditation \& the resulting spiritual force field in individuals (to begin with) on base electromagnetic field produced in the neighbourhood of the practitioner of SuratShabda Yoga. The recorded data can then be studied later to analyze the varying effects of yoga with a view to drawing appropriate conclusions in group setting.

Extending it to Better worldliness can then be made possible, so that the outcome from religion which preaches un-worldliness and science which teaches worldliness can come together in an integrated model 
of "Better worldliness".

\section{References}

[1] Image Source: Global Consciousness Project.

[2] Towards Integration Arts \& Science 2015. Vision Talk by
Most Revered Prof. P.S. Satsangi/Dayalbagh Educational Institute.

[3] Roger Nelson/World Future Society.

[4] Expositions on Truth, Ultimate Reality and Supreme Being, by Most Revered Prof. Satsangi, P. S. (Dayalbagh Educational Institute). 